\title{
Using Spectral Line Profiles as a Diagnostic of the Plasma Electron Density
}

\section{by}

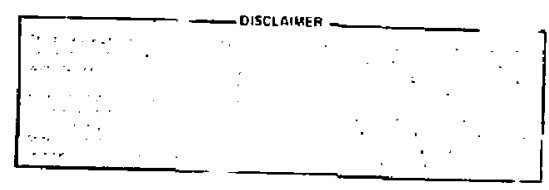

R. W. Lee* and D. L. Matthews

University of California, Lawrence Livermore National Laboratory P.0. Box 5508, Livermore, California 94550

\section{ABSTRACT}

A description of a simple interactive fitting procedure is given which uses line profiles calculated for nighly stripped ions, i.e., hydrogenic, helium-like and lithium-iike species. Infomation on the calculation of these profiles is provided. The experimental data is read from disk, so any appropriately formatted data can be referenced.

*Permanent Address: Spectroscopy, Blackett Laboratory, Imperial College, London, Eng̣land 


\section{Introduction}

The use of spectral-line profiles as a diagnostic tool for plasma electron density has been wel1-documented since the hydrogen $n=2$ to $n=$ 4 transition, $H B$, was first used as a measure of stellar atmospheric electron density. In our case we are interested in more than the hydrogen-line profiles. We attempt to use certain hydrogenic, helium-like and lithium-7ike profiles that are emitted by ions in various plasma sources. Our attention is focussed on the line shape as a function of plasma electron density, $n_{e}$, since if the line emission is optically thin, the fit between the emitted profile and the theoretical curve should yield a measure of the plasma density. Unfortunately, in practice, there are many assumptions which must be proved valid before we can give any clear indication of the accuracy of the $n_{e}$ determination. These assumptions are:

A) The line shape is not dominated by instrument function, i.e., the instrument width $\ll$ intrinsic line width.

B) The temperature of the plasma can be ascertained to a degree of accuracy to ensure that:

1) The temperature variation of the collisional broadening does not inodify the profile, so the same fit is obtained for a wide range of $n_{e}$ within the temperature range, and

2) The thermat Doppler effect should be sfiall compared to the intrinsic width of the line profile. If this is not true, the $l$ ine is a temperature diagnostic. 
c) The line is opticaily thin, so the width, and shape, are functions of local plasma conditions, not the radiation transfer. (For methors where optically thick lines can be used as a diagnostic, sef Appendices).

D) The quality of the data and theory justify the degree of accuracy derivable in the fitting procedure. (For a method of analyzing woisy data see Appendix 1 ).

If these constraints have been met, the line shape with its various features, e.g., slope, width and adjacent components can be an accurate diagnostic. However, in reality, only in experiments specifically designed to do line-shape studies are a!l these requirements met. Therefore, the nature of this fitting procedure is to facilitate line-shape diagnostics in hoth the ideal and normal cases.

\section{General Comments on the Program}

The program, called FIT, and the controlee RUNFIT can be found on .575314 :RWLEE. It is an FTN program and runs compatibly with the line profile programs described in document $U C I 0=18876$. The basic requirements are to have an experimental data set wit,i: two header lines and spectral data, one energy and intensity per line (energy in EV), ano some theoreticaliy calculated profiles (the calculation of which are described in UC10-18876) both on disk. One starts the program, inputs the data interactively, initiates the filting by lining up the two sets of data, and proceeds to $f$ it the profiles by monitoring TMDS frames. This procedure can be repeated $f$ ar new data, either experimental and/or theoretical. The output is a picture $f j l e$ of the fits, which can be viewed using UXTV, and a print file ToJT, which contains hardcopy of all the fitting performed in a run.

\section{Running the Program}

Hote that FTM produces prompts, i.e., a "?", when it wants data; and further, it accepts free format input either space, or conna delimited. 
However, if "iteris are asked fur, $n$ entries must be maue, except when carriage return (denoted CR below), which implies a default is acceptable fur the entire input string.

The program is most easily discusseu in three sections:

A) input

1) Experimental Vata Set (in Detault)

Hen the progret: siarts, the first input is the experimental data set name, which must we nic greater tman 10 characters. This must be conterea; there is no default.

2) lustrumental Line Shape (Deiault hilowea)

The nex input is the in. Lrumerit lidif-molf viutn, in EV, and Live lype of instrument function $1=$ Lorenizian, $0=$ Gáussian. The default, i.t., a Ch response, yitlus a Lureniztian with a llali-llaif wiath ot . U⿺s $\mathrm{EV}$.

3) Profile Information (No Diefaulis)

lne request for input protale information requires tares numbers:

a) Input Profile Nurnber:

Inis is the number cenoting which transition of a particular element is requireo, See Table I for the full list of numbers.

b) Element :

ruis is request for the ion species type; 1 = pyurugenic, $<=$ nelium-like, $j$ = lithium-like. 
Hote: the numuer must correlate correctly with the tneoretical data set (see 1].A.4 be fow), or the input data will be read incorrectly.

c) Ellergy:

This is the lint center energy of the transition of

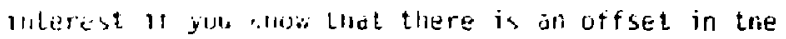
Jula. First illit inrudyn pul U.u, thereafter having seen

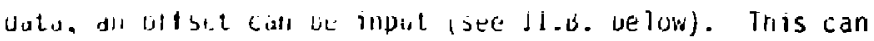
speteo ap prugral., laxecition.

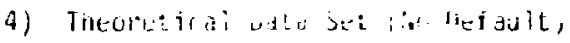

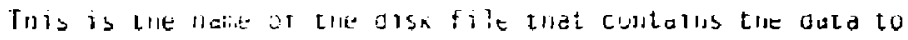
be fil. I l libot ie its inall, ur equal io, iu characters.

\section{3) Initjalizitio;}

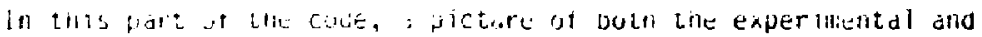

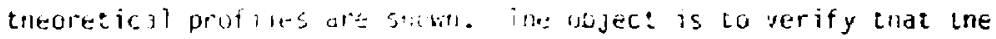

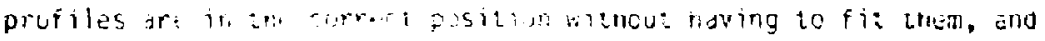

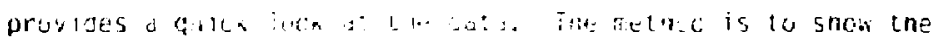

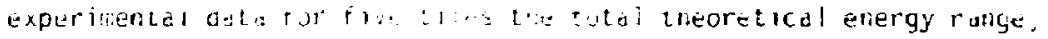

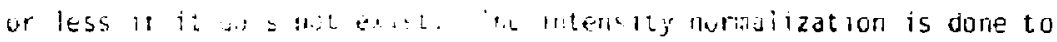

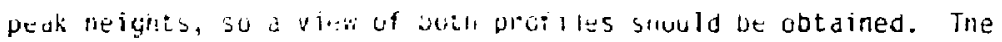

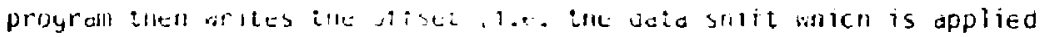
lu the theotoitical energy scale tu uttaill a vest first-pass

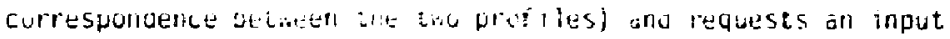
otfset. Tre valiu responses c: $=$

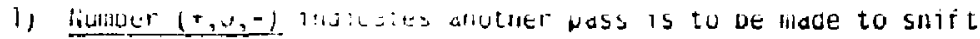
the whule theortical adia set. $\dot{a}+$ numuer tnoves the theory to nigher thergy uj + nubler th. Tile idext response will be a request for a new of ifet. 
2) Ch indicates that the oata are lined up close enough to fit. The next respurise will ue for fitting information (see III. C ve low).

3) -yygy. inuicates a restart with a new theoretical data st:t. Inis can occur when tile present data set is woviously not going to fit (e.g. it is mucn too wiue vihen opzically tnin). The next response will we a request fur profile none isee 11. A. 4).

Note: Tilc uffet can ve used in subsequent fits in trie elleryy input (see 11. H. 3) if a colinun bias is fuluna.

\section{() Fitting}

Once the off set has been determines, the energy of the theoretical data is ccisiutered fixed. Now the program requires information on the detailed fitting of the profiles. The requests in the order of their occurrence and the appropriate responses are listed belo:

1) Request fur 2 and $r / c c$

The irituriation requested siere is the geumetrical path length in cin dio the ground state ion number density of the transition in number/cc. Tuis is used bl a siriple slab-geolitetry optical dept" movel. lout $=8\left(1-t^{-t}\right)$ where $\dot{B}$ is twe black body suarce sunction at the plasma temperature and $r=$ own with o the line profile.

a) CR inaicates tne values snown are acceptable.

b) Input $x$ and $n / c c$.

Note: Do not allow $\ell=0, n / c c=0$ to be used. The program will izil. 
2) Request for Snift

This is attempting to "fine tune" the relative positiuns of the two profiles. Note carefully that these shifts do not permanently shift the data (unlike OFFSET-see 11. H.). The responses are:

a) $+, 0,-$-numbers moves the experimental prufile such tolat

+ numbers move texperimental data to lower energy.

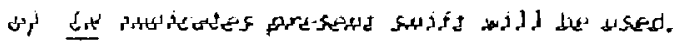

c) -yyyty. inulcates desire tn sigp prograr..

d) gyyy. Huicates desire to input ne: nispoer ror theoretical data ille lext respouse will oe rur profi:. miturtiction (see section li. is. s.).

3) Request fur infuritation on Type of Fiting

There are four possibjlities with the present progran:

a. Scairng tu a given cata point,

1. sreà nurialeirzáliur,

c. iru liogitications, i.e., just plutting and,

d. r least squares fit.

d) + Hulnuer inaicates inat the "th: experimental point, (arrivel di by starting frum the furst experimental puint which overlaps the theory at low energy) will de used to scale the prufiles. Un the plut an "A" will appear at tals point. 
B) Q indicates ihat tne curves will be area normalized by taking onjy the points of averlap.

c) - Number indicates that notning will be done to the data Defore plotting. This is only useful if you believe you nave an LIE radiator of tixed and $\mathrm{n} / \mathrm{Cc}$ and nave ausolute experimenzal uatá sulce $\coprod_{\text {UUT }}^{\text {THEOKY }}=B\left(1-E^{-T}\right)$.

v) Cir inuicates tnat a least squares tit is desirta. In this cast the progran requests infortation concerning wnich overlapped points are to we used. The proyram returns the first and last poirit initially. fue valid responses are:

i) $\mathrm{H}, \mathrm{N}^{\mathrm{l}}:$ (where $\mathrm{N}$, $\mathrm{H}^{\mathrm{l}}$ are two numbers within the range specified) indicates the fit occurs betwean points $N$ and $N^{\text {? }}$.

ii) CR: indicates the points already printed are satisfactory. In tnis rase, the plot will have two "x"s to denute the boundaries of the $f i t$.

4) Request fur intext Step

The prugialit prinls out the temperature, density $k$ and nucc and requests infurmation on the next step. The responses are:

a) "1": inuicates siop program.

u) "L": inaicates another fit with the same cata is desired; the next request will be for shift (see lll. C. C.).

c) "3": inoicates a desire to change aptical deptn; the next request will be for $\ell$ and $n / c c$ (see $I[L . C .1$. ). 
d) "4": indicates a desire to change theoretical data; the next request will de for profile information (see l]l.A.J.).

e) "s": inducates a uesire tu charage experimenial data set; the next reyuest will be for experimental protile nalle (see 11.A.1.1.

f) LR: indicates the sarke as infut " $\angle$ ".

\section{V. Uutputs}

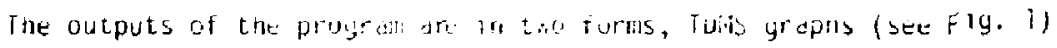
and printout. In acoltun, at ine eno of a portucular run, the yraphics

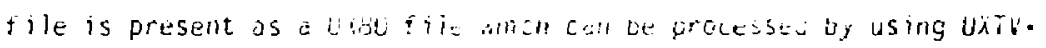

4) Pictures

There are two types of plitures, one for the initialization sequence (see ili.l.) and one for the fiting sequence (see III.C.).

1) Lolitill Pid, F:edi,re:

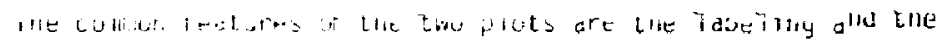

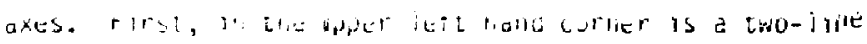

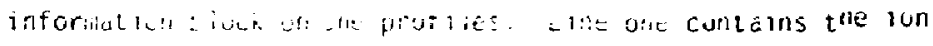

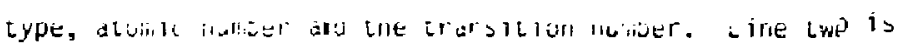
temperature, delsily ailo zll/ci product fur the fit. fopve the plot is one line contaimung inforibation to assist ill estimating tile quality of $i \mathrm{i}$, Llle sinft, the normalized sum of the squares for Gevailon detween the profiles, the experimental nair in

Farther, the adscissa is in $E V$ and the ordinate is in relative intensicy units. 
2) Differences in inc Plots

The differences in trle t:do profiles are the following:

a) The mitialization plut has the experimental data points marked by "x" and condicted sy solid-line segments. Tre trieoretical datd is gerrted ay a dotted line.

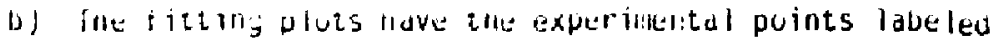
with an "* ara inte takertetical curve is a continuous line. In auditiun, if the scaling or least squares fiting options are chusen ont ur tho "ᄉ"s will appear to denote the points of illterest (see ild.L.S.).

B) Hardcopy

The file TOUT is created fur each run. It is rewound and then written over, so it iust ise bealt with oefure rerunning to be saved. The inforination on the file contains the data set nanes, the temperature and density of the thenretical data, the type of $f i t$ and its paralieters. Inat is, the generalized $f i t$ is given an input protile $I(*)$ and energies $w$. The firal fitted data is

$$
I_{F(T}=A * 1(W-(S)+B
$$

where

$$
\begin{aligned}
& A=\text { sceie factor } \\
& B=\text { continuun level } \\
& \Delta=\text { shift. }
\end{aligned}
$$

Then the experimental data, original theory, the new theoretical data and tne energy are printed. Finally, the half wiuths are printed. 
Appendix 1: Proceaure far Gotaining $\mathrm{n}_{\mathrm{e}}$ from trie wings of a Line.

In cerlain cases, tlle line cures are wot reliavle tither decause the opacity and $\|_{e}$ oenisity are not known or because the line core nas saturated the detectir. system. (See Appendix 2 for cases where the signal-to-noise rativ is iom, but a series of lines are present.)

Smith arld Peacock (J. Pnys of, 11, 2idy, (197y)) have pointed out that if one can ascertain a poillt in the line willg, whlch is known disolutely, then the region sliglitly beyond the half wioth $c$ an be fit and an electron density determined. Essentially, the likthod would be to pick a point in the wing by using the scaling option for a series of different theoretical profiles. Tnis requires a reasonable knowledge of the plasma $Z$, wut this is nut crucial. isee $R$. w. Lee ano A. J. Freenan, JQSRT, 24, 43 (igov) fur the serisitivity af the nicrofielc, which is at question nert, to the perturuer charge. Int iact that one can use tie fill calculaticns to generate the provile onto the tiear line wing is a usetul extension of the tecimiulue. The tits snuuld de accurate tu $20 \%$ if ane can uvtain an assoluie intensiay. 
Appendix 2: Procedures for Ubtaining Plasma Parameter From Series of Lines

In the case where a series of low-signal-to-noise ratio is present, some optically thick and at least one optically thin, the program can be used to find not only the electron density but also the ground state number density, $n_{1}$. The basic concept is to use equal area normalization and ascertain the best fits consistently for all lines.

Although other fitting proceaures such as a least squares fit were tested, it was felt that area normalization maintained the most objective fitting procedure. To characterize the quality of the fit, a normalized suil of the squares paraneter $S$ was used. This $S$ is defined by:

$$
S=\varepsilon\left(\gamma_{\text {exp }}-\gamma_{t h}\right)^{2} \Sigma Y_{t h}{ }^{2}
$$

and is printed out on the top of eacil plot. Here $y_{\exp }$ and $t_{t h}$ are the experimental and theoretical points at the same wavelength, and che sull is over all of the digitized experimental points. In general, a fit witis $Q=.01$ is yood, whereas a fit with $\varphi=0.1$ is poor.

The two major variables in determining the width of the cuserved emission line are $n_{e}$ and $n_{1}$ for a given $\ell$. The gross width of any $l$ ine is not a unique function of $n_{a}$ and $n_{7}$. The details of a line shape, e.g. the height of shoulders, may be a unique function of $n_{e}$ and $n_{1}$, but it is only the coarse features that are observable. If the tneory is compared with given experimental profile, then for smal] optical depth the line width will be a function of $n_{e}$ only, and if the line of best $f$ it is plottea on graph of $n_{e}$ versus $n_{j} l$ as in Fig. 2 , it will be a horizontal line. As the line decomes optically thick, the same total theoretical width can be obtained with a smaller $n_{e}$. Thus, the line of best fit curves cownwards as in Fig. 2. Ideally, a line of vest fit coula be obrained for several transitions, ano they woula

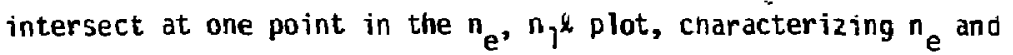
$\mathrm{n}_{1}$ in the siab. 
In fact, the line of fit is an area, determined by the constaint on 4 wrien can be taken as i $<2 \psi_{\min }$ where $\psi_{\min }$ is the quality of the best $f i t$. Thus, instead of a point in $n_{e}, n_{j} l$ space, an area should be defined by the common overlapping area of several transitions.

The maximum number density of ionization the stage of interest can also be represented on the $\mathrm{n}_{\mathrm{e}} \mathrm{n}_{\mathrm{p}}$ plot. $1 \mathrm{f}$, for example, tne plasma is of one elenent of atomic defisity $n_{a}$, ano average charge $z$

$$
n_{h} ; 0.5 n_{a}=\frac{0.3}{2} n_{c}
$$

thus describing an allowed region in the $n_{e}, n_{1}$ parameter space. The end result is fit witil a set of error jounds indicating the quality of the data and the sensitivity of the theory.

The outputs of the progran, both graphical und hardcopy, can oe used as aids in fitting line profiles with this method. 
Appendix 3: Running Job From Source

To obtain the program FIT from storage and run it, one needs to get it from .575374.RWLEE. Then, since it is an FTN program that uses graphic, the graphic file FTNTV80 must be used. To get this use

EXE LASLFTN FTNTVBU DR. / T V

To run the prograll, use the following:

FTN $(I=F I T, L=0, C N A M E=R U N F \perp T, S Y M=S, G L I B=F T N T V S O, G U) / T$

This will create the contrallee Ruplit and start eyecution of the jou. The compilation listing is on " 0 ". 
Appendix 4: ubtaining Hardcopy of Plots

In general, the plot files wil' be UX files with names lin: UALFTHudiou. The files can de processed in any fianner, nowever, the entire $U X$ file prucessing system is not well-d.sumented. Here are two simple choices:

1) UXTV will snow the pictures dgain.

a) Uxhu will send theili to an kut. The mput line here is:

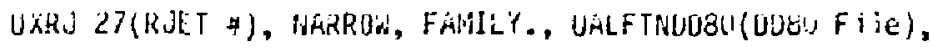
Hufll, iUuifi. . $135 \mathrm{miLL}$.

The graphs will then $r$ it on ane versatec page. 
Tanle 1 - Key to Input

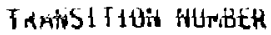

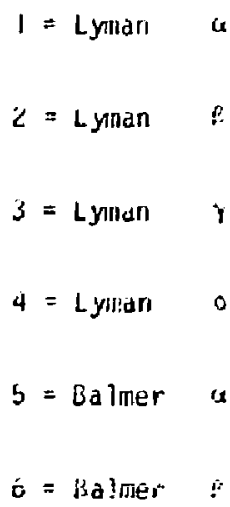

$1=1 s^{2} I_{S_{0}}-1 \sin ^{1} p$

$2=15^{2} t_{0}-15 \sin ^{3} \mathrm{P}$

$s=i s^{2} s_{0}-154 p^{3} p$
ELEHEHT HUMPER

1 = Hydrogenic

$\dot{L}=$ rielium-like 


$$
\begin{aligned}
& i=25-7 \\
& z=6^{n}: \pi^{35} \\
& 3=4 r^{\prime} 3 / 2^{-3 i} \\
& A=\left\langle P{ }_{1 / C^{-S t}}\right. \\
& D=c P 1 / x^{-3 t} \\
& 0=3-4 \\
& \Sigma=2,[11 \mathrm{um}-] \mathrm{ike} \\
& I=\left\langle H_{3} H^{-}\right. \\
& \ddot{*}=2 \mathrm{P} s / c^{-40} \\
& y=\mu H_{i / c^{-\infty}} \\
& 111=\angle P B / e^{-41}
\end{aligned}
$$


SAIPLE TMDS IRAPH

Intensity

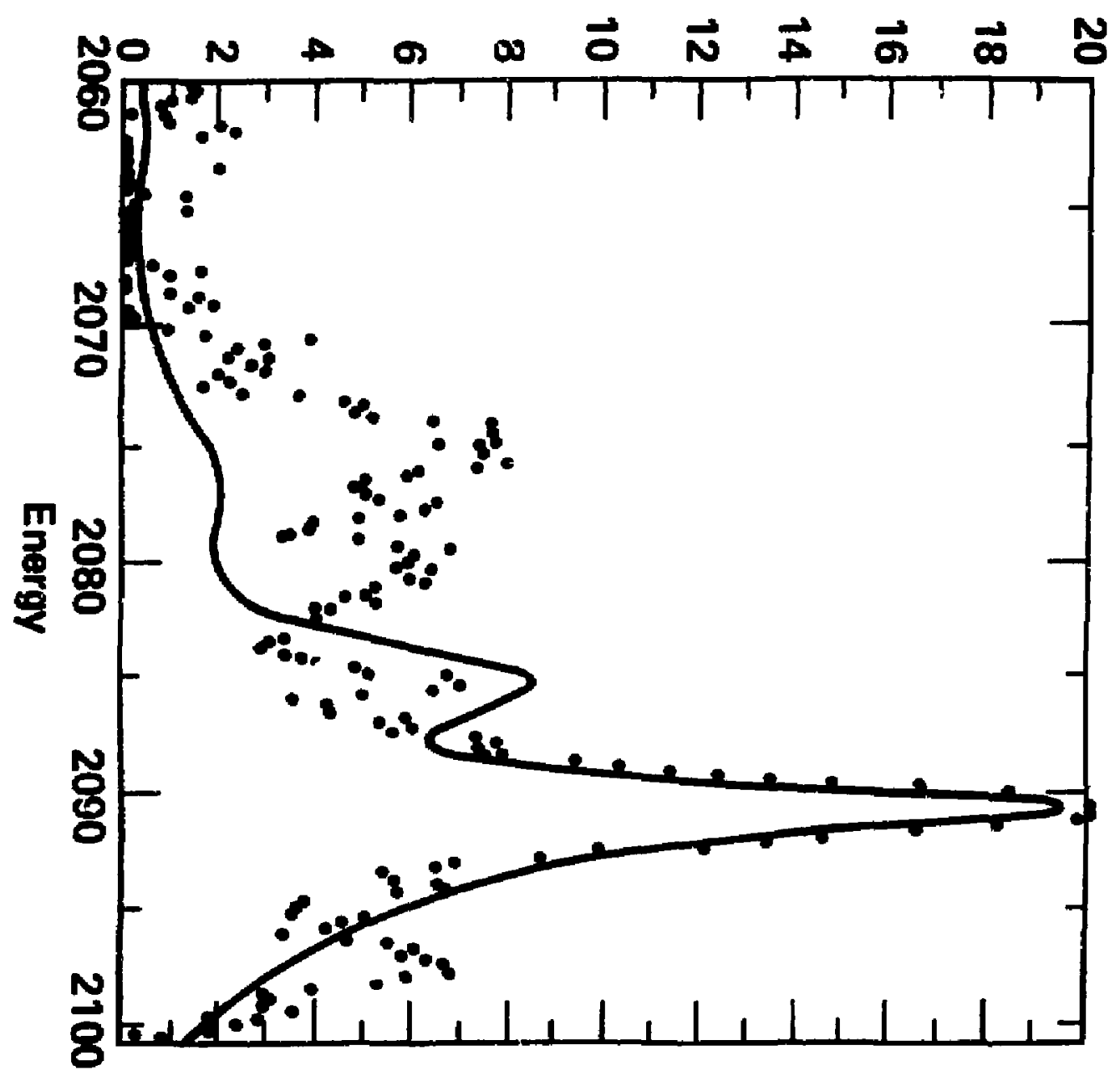

Figure 1 


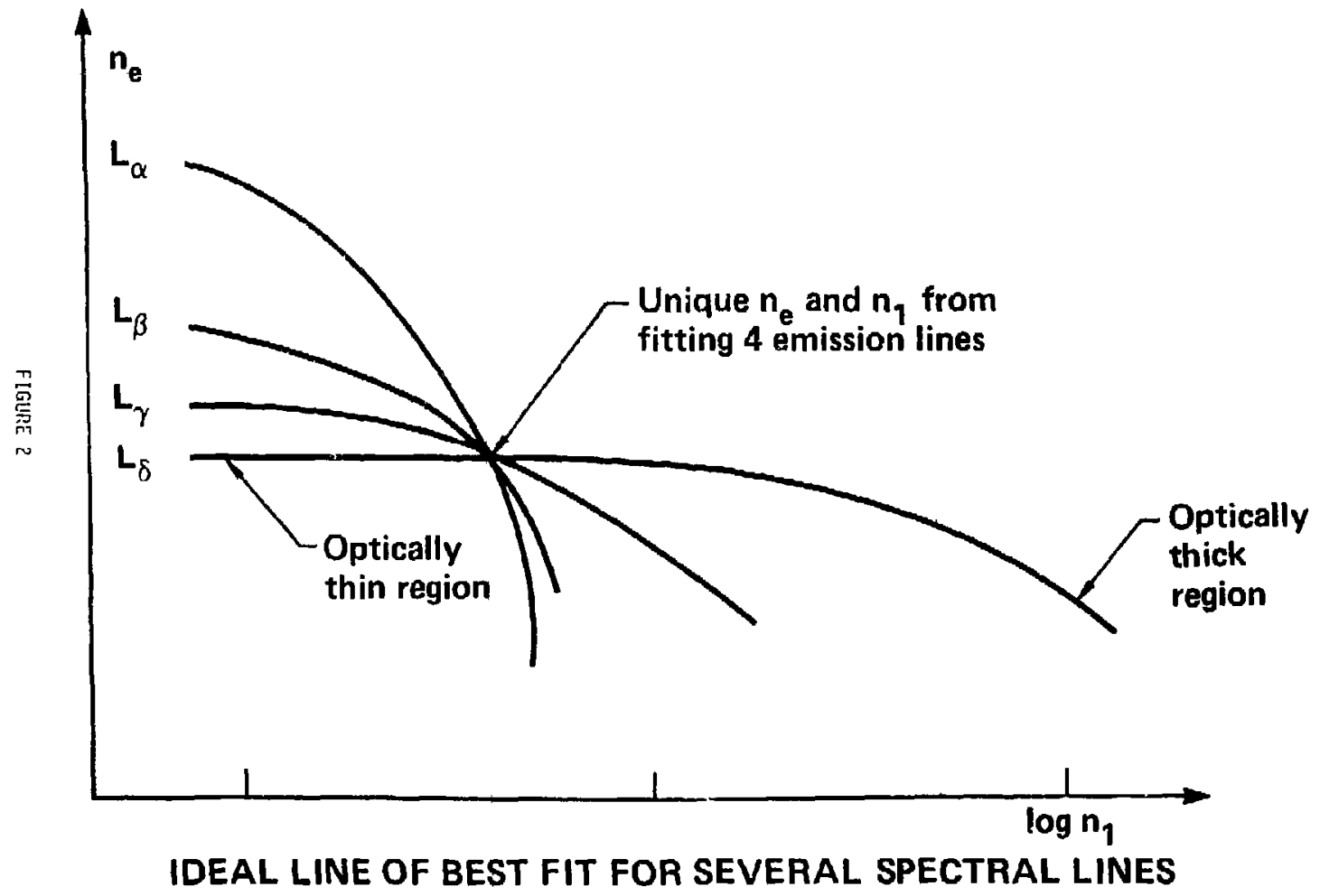


SAMPLE EXPERIMEITT INPUT FILE

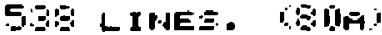

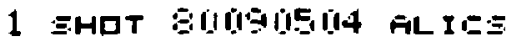

ENATEST FCER

$\because \quad \Xi$. OEE

4 E .

1. 1. بE +

5 E. 10E.

9. $09301011 \mathrm{E}+0 \mathrm{~B}$

E. E.

$\therefore 40545.54+11 E$

$7 \quad E .07 \%$

B. $17 \% 1545 F+10$

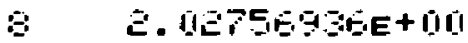

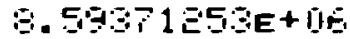

G 2 . 1

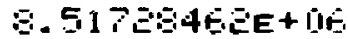

10

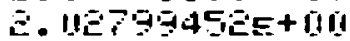

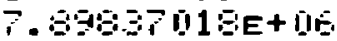

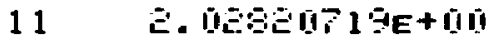

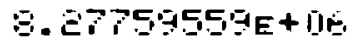

$1 \overline{2}$

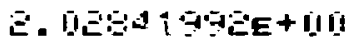

E. $174714 E E+I I E$.

$13 \quad$ E. IE

$7.17470195 E+11 \%$

$14 \quad \Xi-10384555 E+10$

5 .

$15 \quad 2.0100150+110$

5 -

$15 \quad$ E. 1

4.

17

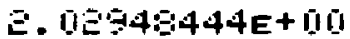

4. $7453713 E+11 E$

$1:$

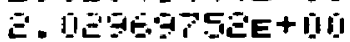

4 . 4 11EESEETE

19

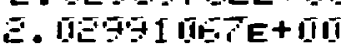

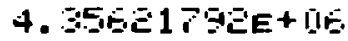

E)

E. 11

4. 1TI TFIE+ IE

$\because 4101551 E+016$

...ETI 


\section{SAMPLE PROGRAM EXECUTION}

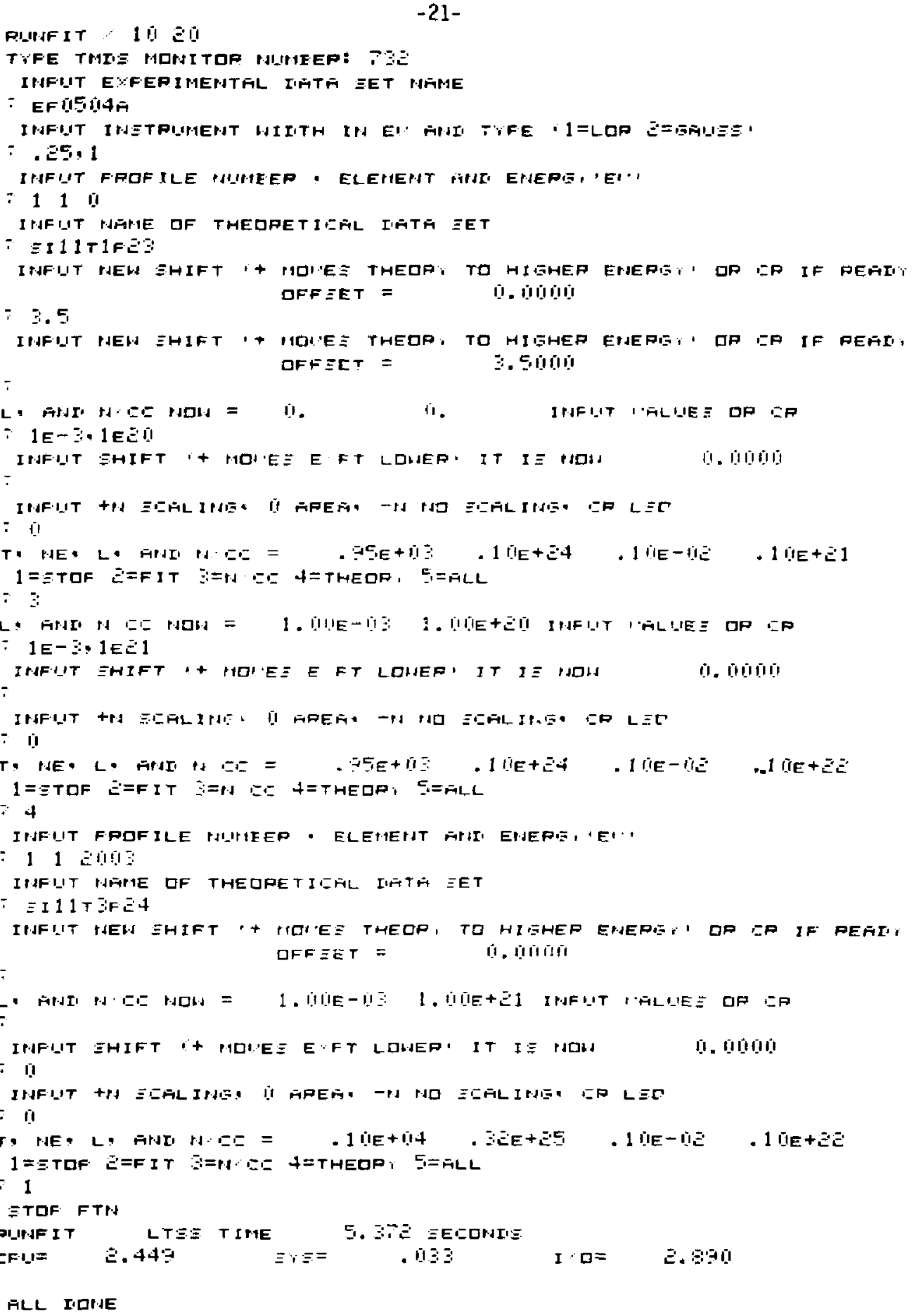

